\title{
EMBEDDING SOME BORDERED RIEMANN SURFACES IN THE AFFINE PLANE
}

\author{
Miran Černe AND Franc Forstnerič
}

\section{Introduction}

It is a long-standing open problem whether every open (non-compact) Riemann surface $\mathcal{R}$ admits a proper holomorphic embedding into $\mathbb{C}^{2}$. The most general result in this direction was proved by Globevnik and Stensønes [GS]: Every finitely connected domain in $\mathbb{C}$ with no isolated points in the boundary embeds in $\mathbb{C}^{2}$. Earlier constructions of embeddings in $\mathbb{C}^{2}$ are due to Kasahara and Nishino for the disc $U=\{z \in \mathbb{C}:|z|<1\}$ [Ste], Laufer [Lau] for the annuli $A=\{1<|z|<r\}$ and Alexander [Ale] for the punctured disc $U \backslash\{0\}$. Every open Riemann surface embeds in $\mathbb{C}^{3}[\mathrm{GR}]$, and every compact Riemann surface embeds in $\mathbb{C P}^{3}$ but most of them don't embed in $\mathbb{C P}^{2}[\mathrm{FK}]$.

In this paper we consider the embedding problem for bordered Riemann surfaces. The underlying space is a compact, orientable, smooth real surfaces $\mathcal{R}$ with boundary $b \mathcal{R}=\cup_{j=1}^{m} C_{j}$ consisting of finitely many curves. Topologically $\mathcal{R}$ is equivalent to a sphere with $g_{\mathcal{R}}$ handles (the genus of $\mathcal{R}$ ) and $m \geq 1$ discs removed. A complex structure on $\mathcal{R}$ is determined by a real endomorphism $J$ of the tangent bundle $T \mathcal{R}$ satisying $J^{2}=-$ Id (Gauss-Ahlfors-Bers; for higher dimensions see Newlander and Nirenberg [NN]). Two complex structures $J_{0}$ and $J_{1}$ are equivalent if there exists a diffeomorphism $\phi: \mathcal{R} \rightarrow \mathcal{R}$ satisfying $d \phi \circ J_{0}=J_{1} \circ d \phi$. The set of equivalence classes of complex structure on $\mathcal{R}$, denoted $\mathcal{M}(\mathcal{R})$, is called the moduli space of Riemann surface structures on $\mathcal{R}$. We emphasize that we do not deal with punctured surfaces, i.e, $\mathcal{R}$ has no isolated points in the boundary.

Our first result is that the topology of a bordered surface plays no role in the problem of holomorphic embeddability in $\mathbb{C}^{2}$.

Theorem 1.1. On each bordered surface $\mathcal{R}$ there exists a complex structure such that the open Riemann surface $\mathcal{R}=\mathcal{R} \backslash b \mathcal{R}$ admits a proper holomorphic embedding in $\mathbb{C}^{2}$.

We give an elementary proof of Theorem 1.1 in section 2 . In fact we will show that there is a non-empty open set of non-equivalent complex structures on $\mathcal{R}$ for which $\mathcal{R}$ embeds into $\mathbb{C}^{2}$ (Theorem 1.5).

The precise regularity of $\mathcal{R}$ up to the boundary is not important since $\mathcal{R}$ is biholomorphically equivalent to a domain bounded by $m \geq 1$ real-analytic

Received August 7, 2001. 
curves in a compact Riemann surface $\widetilde{\mathcal{R}}$. The simplest way to obtain such $\widetilde{\mathcal{R}}$ is to fill each hole of $\mathcal{R}$ by attaching a disc $D_{j}$ such that we identify $b D_{j}$ with a boundary curve $C_{j} \subset b \mathcal{R}$ and extend the complex structure across $C_{j}$ using reflection. Another possibility is to embed $\mathcal{R}$ in the (Schottky) double $\widehat{\mathcal{R}}$ which is obtained by gluing two copies of $\mathcal{R}$ (with the opposite orientation) along $b \mathcal{R}$. The genus of $\widehat{\mathcal{R}}$ equals $2 g_{\mathcal{R}}+m-1$. The details of this 'doubling construction' can be found in [BS, pp. 581-2], [Spr, p. 217] or [SS]. Hence we shall assume from now on that $\mathcal{R}$ is smooth up to the boundary.

We shall now give a more precise embedding theorem. For each $k \geq 0$ we denote by $\mathcal{A}^{k}(\mathcal{R})$ the algebra of all $\mathcal{C}^{k}$-functions on $\mathcal{R}$ which are holomorphic in $\stackrel{\circ}{\mathcal{R}}$. A nonconstant function $f \in \mathcal{A}(\mathcal{R})$ satisfying $|f|=1$ on $b \mathcal{R}$ is called an inner function on $\mathcal{R}$. The restriction of an inner function to $\mathcal{R}$ is a proper holomorphic map of $\mathcal{R}$ onto the disc $U$; conversely, any proper holomorphic map

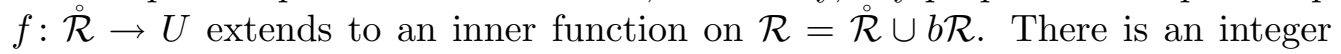
$d=\operatorname{deg}(f) \in \mathbb{N}$, called the degree (or multiplicity) of $f$, such that for all except finitely many points $z \in \bar{U}$ the fiber $\mathcal{R}_{z}=f^{-1}(z)$ consists of $d$ distinct points in $\mathcal{R}$ while the exceptional fibers consist of less than $d$ points.

Definition 1. A bordered Riemann surface $\mathcal{R}$ of genus $g_{\mathcal{R}}$ and with $m$ boundary components is said to be of class $\mathcal{F}$ if it admits an injective immersion $F=$ $(f, g): \mathcal{R} \rightarrow \bar{U} \times \mathbb{C}$ which is holomorphic in $\stackrel{\mathcal{R}}{ }$ and such that $f$ is an inner function with $\operatorname{deg}(f) \geq 2 g_{\mathcal{R}}+m-1$.

Clearly this property is biholomorphically invariant. The reason for introducing this class is the following result which is proved in section 3.

Theorem 1.2. If $\mathcal{R}$ is a bordered Riemann surface of class $\mathcal{F}$ then $\mathcal{R}=\mathcal{R} \backslash b \mathcal{R}$ admits a proper holomorphic embedding in $\mathbb{C}^{2}$.

\section{Examples.}

1. On each smoothly bounded domain $\Omega \subset \subset \mathbb{C}$ with $m$ boundary components there exists an inner function $f$ with $\operatorname{deg}(f)=m$ [Ahl]. The map $F(x)=$ $(f(x), x) \in \mathbb{C}^{2}$ for $x \in \bar{\Omega}$ satisfies the hypothesis of Theorem 1.2 and hence $\Omega$ embeds in $\mathbb{C}^{2}$. This is the theorem of Globevnik and Stensønes [GS].

2. A compact Riemann surface is called hyperelliptic if it is the normalization of a complex curve in $\mathbb{C P}^{2}$ given by $w^{2}=\Pi_{j=1}^{k}\left(z-z_{j}\right)$ for some choice of points $z_{1}, \ldots, z_{k} \in \mathbb{C}[\mathrm{FK}]$. We shall call a bordered Riemann surface $\mathcal{R}$ hyperelliptic if its double $\widehat{\mathcal{R}}$ is hyperelliptic. Such $\mathcal{R}$ has either one or two boundary components and it admits a pair of inner functions $(f, g)$ which embed $\mathcal{R}$ in the polydisc $U^{2}$ such that $b \mathcal{R}$ is mapped to the torus $(b U)^{2}$; moreover, one of the two functions has degree $2 g_{\mathcal{R}}+m$ and the other one has degree 2 (see [Ru1] and sect. 2 in [Gou]). Thus $\mathcal{R}$ is of class $\mathcal{F}$ and we get:

Corollary 1.3. If $\mathcal{R}$ is a hyperelliptic bordered Riemann surface then $\mathcal{R}$ admits a proper holomorphic embedding in $\mathbb{C}^{2}$. In particular, each torus with one hole embeds properly holomorphically into $\mathbb{C}^{2}$. 
Indeed it is shown in [Gou] that the double $\widehat{\mathcal{R}}$ of a hyperelliptic bordered Riemann surface $\mathcal{R}$ can be represented as a curve in $\mathbb{C P}^{2}$ given by the equation

$$
y^{2}=\prod_{j=1}^{\hat{g}+1}\left(x-\alpha_{j}\right)\left(1-\overline{\alpha_{j}} x\right)
$$

for some choice of distinct points $\alpha_{j} \in U(1 \leq j \leq \hat{g}+1)$, where $\hat{g}=2 g_{\mathcal{R}}+m-1$ is the genus of $\widehat{\mathcal{R}}$, such that $\mathcal{R}=\{(x, y) \in \widehat{\mathcal{R}}:|x| \leq 1\}$. The pair of inner functions on $\mathcal{R}$ given by $f=y / \prod_{j=1}^{\hat{g}+1}\left(1-\overline{\alpha_{j}} x\right), g=x$, provides an embedding $F=(f, g): \mathcal{R} \rightarrow(\bar{U})^{2}$. Clearly $g$ has multiplicity 2 on $\mathcal{R}$. From the relation $f^{2}=$ $\prod_{j=1}^{\hat{g}+1}\left(g-\alpha_{j}\right) /\left(1-\overline{\alpha_{j}} g\right)$ which follows from (1.1) we see that $f$ has multiplicity $\hat{g}+1=2 g_{\mathcal{R}}+m$ and hence Theorem 1.2 applies. Sikorav [Sik] gave a slightly different proof of Corollary 1.3 for tori with one hole (unpublished); these are all hyperelliptic.

Sketch of the proof of Theorem 1.2. Let $P \subset \subset \mathbb{C}^{2}$ be a polydisc. According to [Glo] (see also [C̆G] and [Stn]) there exist Fatou-Bieberbach domains $\Omega \subset \mathbb{C}^{2}$ such that $b \Omega \cap P$ is an arbitrarily small perturbation of the cylinder $(b U \times \mathbb{C}) \cap P$ (Proposition 2.1 below). Such domains $\Omega$ can be constructed using sequences of compositions of shears in coordinate directions on $\mathbb{C}^{2}$. Let $\phi: \Omega \rightarrow \mathbb{C}^{2}$ be a biholomorphic map onto $\mathbb{C}^{2}$. If $F=(f, g): \mathcal{R} \rightarrow \bar{U} \times \mathbb{C}$ is as in Theorem 1.2, we choose the polydisc $P$ large enough to contain $F(\mathcal{R})$ and solve a Riemann-Hilbert boundary value problem to find a new holomorphic embedding $\widetilde{F}=(\widetilde{f}, g): \mathcal{R} \rightarrow$ $\bar{\Omega} \cap P$ such that $\widetilde{F}(b \mathcal{R}) \subset b \Omega$. The map $G=\phi \circ \widetilde{F}: \stackrel{\mathcal{R}}{\rightarrow} \mathbb{C}^{2}$ is then a proper holomorphic embedding of $\mathcal{R}$ to $\mathbb{C}^{2}$. The details are carried out in section 3 . This approach was used in [ČG] for finitely connected planar domains to provide a different proof of the embedding theorem of Globevnik and Stensønes [GS]. The proof of Theorem 1.1 (Section 2) is similar but more elementary.

The proof of Theorem 1.1 shows that each bordered surface $\mathcal{R}$ carries a complex structure $J$ such that $(\mathcal{R}, J)$ is of class $\mathcal{F}$. We will show that the set of such complex structures on $\mathcal{R}$ is open. To be precise, fix a number $\alpha \in(0,1)$ and denote by $\operatorname{End}_{\mathbb{R}}^{\alpha}(T \mathcal{R})$ the set of all endomorphisms of $T \mathcal{R}$ which are Hölder continuous of class $\mathcal{C}^{\alpha}$, endowed with the $\mathcal{C}^{\alpha}$ topology. Let

$$
\mathcal{J}_{\mathcal{R}}^{\alpha}=\left\{J \in \operatorname{End}_{\mathbb{R}}^{\alpha}(T \mathcal{R}): J^{2}=-\operatorname{Id}\right\} .
$$

Theorem 1.4. Let $\mathcal{R}$ be a smooth bordered surface and $0<\alpha<1$. The set $\mathcal{F}_{\mathcal{R}}^{\alpha}$, consisting of all $J \in \mathcal{J}_{\mathcal{R}}^{\alpha}$ such that the Riemann surface $(\mathcal{R}, J)$ is of class $\mathcal{F}$, is open in $\mathcal{J}_{\mathcal{R}}^{\alpha}$.

Theorem 1.4 is proved in Section 4. The main point is that inner functions on $(\mathcal{R}, J)$ with multiplicity at least $2 g_{\mathcal{R}}+m-1$ are stable under small perturbations of the complex structure $J$ (Proposition 4.1).

For each $\alpha \in(0,1)$ we can realize the moduli space $\mathcal{M}(\mathcal{R})$ as the quotient $\mathcal{M}(\mathcal{R})=\mathcal{J}_{\mathcal{R}}^{\alpha} / \sim$ where $J_{0}, J_{1} \in \mathcal{J}_{\mathcal{R}}^{\alpha}$ satisfy $J_{0} \sim J_{1}$ if and only if there exists a diffeomorphism $\phi: \mathcal{R} \rightarrow \mathcal{R}$ of class $\mathcal{C}^{1, \alpha}$ with $d \phi \circ J_{0}=J_{1} \circ d \phi$. Let $\pi: \mathcal{J}_{\mathcal{R}}^{\alpha} \rightarrow \mathcal{M}(\mathcal{R})$ 
denote the quotient projection. We endow $\mathcal{M}(\mathcal{R})$ with the quotient topology. The set $\mathcal{F}(\mathcal{R})=\pi\left(\mathcal{F}_{\mathcal{R}}^{\alpha}\right) \subset \mathcal{M}(\mathcal{R})$ consists of moduli of Riemann surface structures of class $\mathcal{F}$; this makes sense since the property $\mathcal{F}$ is biholomorphically invariant and therefore $\mathcal{F}_{\mathcal{R}}^{\alpha}=\pi^{-1}(\mathcal{F}(\mathcal{R}))$. We can now summarize the above results as follows.

Theorem 1.5 (Main Theorem). Let $\mathcal{M}(\mathcal{R})$ denote the moduli space of Riemann surface structures on a smooth bordered surface $\mathcal{R}$. The set $\mathcal{F}(\mathcal{R}) \subset$ $\mathcal{M}(\mathcal{R})$, consisting of structures of class $\mathcal{F}$, is nonempty and open in $\mathcal{M}(\mathcal{R})$. For each $[J] \in \mathcal{F}(\mathcal{R})$ the open Riemann surface $(\mathcal{R}, J)$ admits a proper holomorphic embedding in $\mathbb{C}^{2}$. If $\mathcal{R}$ is a finitely connected domain in $\mathbb{C}$ then $\mathcal{F}(\mathcal{R})=\mathcal{M}(\mathcal{R})$.

The last statement above is the theorem of [GS]. The main question is whether $\mathcal{F}(\mathcal{R})=\mathcal{M}(\mathcal{R})$ for every bordered surface $\mathcal{R}$. The discussion in section 2 seems to indicate a negative answer; see Proposition 2.2 and the remark following its proof.

Comments regarding class $\mathcal{F}$. It is proved in [Ahl, pp. 124-126] that on every bordered Riemann surface $\mathcal{R}$ of genus $g_{\mathcal{R}}$ with $m$ boundary components there is an inner function $f$ with multiplicity $2 g_{\mathcal{R}}+m$ (although the so-called Ahlfors functions may have smaller multiplicity). A generic choice of $g \in A^{1}(\mathcal{R})$ gives an immersion $F=(f, g): \mathcal{R} \rightarrow \bar{U} \times \mathbb{C}$ with at most finitely many double points (normal crossings). The main difficulty is to find $g$ such that $F=(f, g)$ is injective on $\mathcal{R}$. We do not know whether such $g$ always exists as Oka's principle does not apply in this situation (Proposition 2.2).

It would be of interest to relax the condition in Theorem 1.2 that one of the components be an inner function. In this direction we pose the following.

Problem: Let $\mathcal{R}$ be a bordered Riemann surface and let $F=(f, g): \mathcal{R} \rightarrow \mathbb{C}^{2}$ be a holomorphic embedding whose image $F(\mathcal{R})$ is polynomially convex in $\mathbb{C}^{2}$. Does $\mathcal{R}$ embed (properly holomorphically) in $\mathbb{C}^{2}$ ?

A possible approach would be to use sequences of automorphisms of $\mathbb{C}^{2}$ to carry the boundary points of $F(b \mathcal{R})$ towards infinity.

The problem of holomorphic embeddability of a bordered Riemann surface $\mathcal{R}$ in $\mathbb{C}^{2}$ is related to the question whether certain algebras of holomorphic functions on $\mathcal{R}$ are doubly generated. If $F=(f, g): \mathcal{R} \rightarrow \mathbb{C}^{2}$ is an $\mathcal{A}^{k}$-embedding whose image $F(\mathcal{R})$ is polynomially convex then $f$ and $g$ generate a dense subalgebra of $\mathcal{A}^{k}(\mathcal{R})$; in such case we say that the algebra is doubly generated. Conversely, if $f$ and $g$ generate a dense subalgebra in $\mathcal{A}^{k}(\mathcal{R})$ then $F=(f, g): \mathcal{R} \rightarrow \mathbb{C}^{2}$ is an injective immersion (not necessarily proper). The question whether $\mathcal{A}(\mathcal{R})$ is always doubly generated is to our knowledge still open. In 1978 Tsanov [Tsa] proved that for any bordered surface $\mathcal{R}$ there is a non-empty open set in the Teichmüller space $\mathcal{T}(\mathcal{R})$ (or in the reduced Teichmüller space $\mathcal{T}^{\#}(\mathcal{R})$ ) consisting of Riemann surfaces for which the algebra $\mathcal{A}^{0}(\mathcal{R})$ is doubly generated. In view of the above remark this also follows from Theorem 1.5. 
Higher dimensional analogues of open Riemann surfaces are Stein manifolds. A complex manifold is Stein if it admits a proper holomorphic embedding in some complex Euclidean space $\mathbb{C}^{N}$ [GR, Hör]. It is known that every Stein manifold of complex dimension $n \geq 2$ admits a proper holomorphic embedding into $\mathbb{C}^{N}$ with $N=[3 n / 2]+1$ and this $N$ is in general the smallest possible [EG, Sch]. For $n=1$ this would give $N=2$; however, the proof, which is based on the 'removal of singularities' method, does not apply for $n=1$ since its main ingredient breaks down. This crucial ingredient is the homotopy principle, also called the Oka-Grauert principle, for sections of holomorphic vector bundles over Stein manifolds which avoid certain complex analytic subvariety of the total space [Gra, Gro2, FP1, FP2, FP3]. When $n=1$, the subset to be avoided is a complex hypersurface and the Oka-Grauert principle fails in general (Proposition $2.2)$.

\section{Proof of Theorem 1.1}

In this section we outline an approach to construct embeddings of bordered Riemann surfaces $\mathcal{R}$ in the tube $\bar{U} \times \mathbb{C}$ by the 'elimination of singularities' method which has been used successfuly in higher dimensions [EG, Sch]. Although we cannot prove the existence of such an embedding for every complex structure on a given surface $\mathcal{R}$, we obtain an elementary proof of Theorem 1.1 based on the following result of Globevnik [Glo]. We quote the version proved in [ČG]. As before, $U$ denotes the unit disc in $\mathbb{C}$ and $r U=\{|z|<r\}$. We denote the coordinates on $\mathbb{C}^{2}$ by $(z, w)$. Fix $r>0$ and let $P=(2 U) \times(r U) \subset \mathbb{C}^{2}$.

Proposition 2.1 ([ČG], Lemma 2.1). There exist arbitrarily small $\mathcal{C}^{3}$-perturbations $S$ of $b U \times \mathbb{C}$ such that, if $\Omega$ is the domain in $P$ bounded by $S \cap P$ and by $|w|=r$, there is an injective holomorphic map $\phi: \Omega \rightarrow \mathbb{C}^{2}$ such that $\left|\phi\left(z_{n}, w_{n}\right)\right| \rightarrow+\infty$ whenever $\left(z_{n}, w_{n}\right) \in \Omega$ and $\lim _{n \rightarrow \infty} \operatorname{dist}\left\{\left(z_{n}, w_{n}\right), S\right\}=0$.

Remark. In fact there exist Fatou-Bieberbach domains $\Omega \subset \mathbb{C}^{2}$ with smooth boundary such that $b \Omega \cap P$ is a small perturbation of $(b U \times \mathbb{C}) \cap P$ (Stensønes [Stn]; see also Globevnik [Glo] for the $\mathcal{C}^{1}$ version). The weaker result quoted above will suffice for our purposes.

Proof of Theorem 1.1. We may assume that $\mathcal{R}$ is a compact domain with smooth real-analytic boundary in a Riemann surface $\widetilde{\mathcal{R}}$ (sect. 1). We denote by $\mathcal{O}(\mathcal{R})$ the algebra of functions $f$ holomorphic in a neighborhood $V_{f}$ of $\mathcal{R}$ in $\widetilde{\mathcal{R}}$. By Ahlfors [Ahl] there is an inner function $f$ on $\mathcal{R}$. Let $Z=\left\{z_{1}, z_{2}, \ldots, z_{p}\right\} \subset \bar{U}$ be the set of critical values of $f$. By the Hopf lemma we have $d f_{x} \neq 0$ for $x \in b \mathcal{R}$ and hence $Z$ is contained in the open disc $U$. Choose a function $g_{1} \in \mathcal{O}(\mathcal{R})$ such that

a) $g_{1}$ separates points on the (finitely many) fibers $\mathcal{R}_{z}$ for $z \in Z$, and

b) $d g_{1} \neq 0$ at each point $x \in f^{-1}(Z) \subset \mathcal{R}$.

Clearly $g_{1}$ will separate points on all except perhaps finitely many fibers $\mathcal{R}_{z}$ $(z \in \bar{U})$. Condition (b) insures that $F_{1}=\left(f, g_{1}\right): \mathcal{R} \rightarrow \bar{U} \times \mathbb{C}$ is an immersion. 
A generic choice of $g_{1}$ also insures that $F_{1}$ has only finitely many double points in $\mathcal{R}$ and no double point on $b \mathcal{R}$. Now choose $g_{2} \in \mathcal{O}(\mathcal{R})$ which vanishes to second order at each point of $f^{-1}(Z)$ and such that the pair $\left(g_{1}, g_{2}\right)$ separates points on all fibers $\mathcal{R}_{z}$ for $z \in \bar{U}$; clearly such $g_{2}$ exists since we must satisfy the separation condition only at finitely many points.

We wish to find $g \in \mathcal{O}(\mathcal{R})$ such that $F=(f, g): \mathcal{R} \rightarrow \bar{U} \times \mathbb{C}$ is an embedding. As in [EG] and [Sch] we seek $g$ in the form

$$
g(x)=g_{1}(x)+\alpha(f(x)) g_{2}(x), \quad x \in \mathcal{R},
$$

where $\alpha: \bar{U} \rightarrow \mathbb{C}$ is a holomorphic function to be selected. Since $g_{2}$ vanishes to second order at each point $x \in f^{-1}(Z)$, we have $g(x)=g_{1}(x)$ and $d g_{x}=\left(d g_{1}\right)_{x}$ at such points. Thus for any choice of $\alpha$ the associated map $F=(f, g): \mathcal{R} \rightarrow \bar{U} \times \mathbb{C}$ is a holomorphic immersion which is injective in a neighborhood of $f^{-1}(Z)$.

Our goal is to choose $\alpha$ such that $F$ is injective globally on $\mathcal{R}$. To formulate the relevant condition on $\alpha$ we fix a point $z \in \bar{U}$ and write $f^{-1}(z)=\left\{x_{1}, \ldots, x_{d}\right\}$ (distinct points!), where $d=\operatorname{deg}(f)$ for all except finitely many $z \in \bar{U}$. Denote by $\Sigma_{z} \subset \mathbb{C}$ the (finite) set of solutions of the equations

$$
g_{1}\left(x_{i}\right)+a g_{2}\left(x_{i}\right)=g_{1}\left(x_{j}\right)+a g_{2}\left(x_{j}\right), \quad 1 \leq i<j \leq d .
$$

Equivalently, a number $a \in \mathbb{C}$ belongs to $\Sigma_{z}$ if it solves the equation

$$
a\left(g_{2}\left(x_{j}\right)-g_{2}\left(x_{i}\right)\right)=g_{1}\left(x_{i}\right)-g_{1}\left(x_{j}\right)
$$

for some $i \neq j$. By the choice of $g_{1}$ and $g_{2}$ at least one of the differences above is nonzero for each pair of indices $i, j$ and hence each equation has either one solution or no solutions. The set $\Sigma=\cup_{z \in \bar{U}}\{z\} \times \Sigma_{z} \subset \bar{U} \times \mathbb{C}$ is a closed onedimensional complex analytic subset of $\bar{U} \times \mathbb{C}$. The function $g$ determined by $\alpha$ according to (2.1) separates the points on all fibers of $f$ if and only if the graph of $\alpha$ avoids $\Sigma$, that is, if $\alpha(z) \notin \Sigma_{z}$ for all $z \in \bar{U}$.

Choose a simple smooth $\operatorname{arc} C \subset U$ containing the set $Z$ of critical values of $f$. By dimension reasons there is a smooth function $\alpha_{0}: C \rightarrow \mathbb{C}$ whose graph over $C$ avoids $\Sigma$. We can approximate $\alpha_{0}$ uniformly on $C$ by holomorphic polynomials $\alpha$. If the approximation is sufficiently close, the graph of $\alpha$ will avoid $\Sigma$ over an open set $V \subset \subset U$ containing $C$. If $g$ is the corresponding function (2.1) then $F=(f, g)$ is a proper holomorphic embedding of $\mathcal{R}_{V}=f^{-1}(V) \subset \mathcal{R}$ to $V \times \mathbb{C}$.

Choose a simply connected closed domain $D_{0} \subset \subset V$ with real-analytic boundary and containing the $\operatorname{arc} C$ in its interior. There are a domain $V_{1} \subset V$ containing $D_{0}$ and an injective holomorphic map $\sigma: V_{1} \rightarrow \mathbb{C}$ which maps $D_{0}$ conformally onto $\bar{U}$. The map $F^{\prime}=(\sigma \circ f, g): f^{-1}\left(V_{1}\right) \rightarrow \mathbb{C}^{2}$ is a holomorphic embedding which maps the closed domain $\mathcal{R}_{0}=f^{-1}\left(D_{0}\right) \subset \mathcal{R}$ to $\bar{U} \times \mathbb{C}$ and it maps $b \mathcal{R}_{0}$ to $b U \times \mathbb{C}$.

Choose a number $r>\sup \{|g(x)|: x \in \mathcal{R}\}$. Let $\Omega$ be as in Proposition 2.1 such that $b \Omega \cap(2 U \times r U)$ is a small $\mathcal{C}^{3}$-perturbation of the cylinder $b U \times r U$. If the approximation is sufficiently good then $b \Omega$ intersects the image of $F^{\prime}$ transversely and the set

$$
\mathcal{R}^{\prime}=\left\{x \in f^{-1}\left(V_{1}\right): F^{\prime}(x) \in \Omega\right\} \subset \subset \stackrel{\circ}{\mathcal{R}}
$$


is a domain in $\stackrel{\circ}{\mathcal{R}}$ with $\mathcal{C}^{3}$-boundary which is a small $\mathcal{C}^{3}$-perturbation of $b \mathcal{R}_{0}$. If $\phi: \Omega \rightarrow \mathbb{C}^{2}$ is chosen as in Proposition 2.1 then the map $G=\phi \circ F^{\prime}: \mathcal{R}^{\prime} \rightarrow \mathbb{C}^{2}$ is a proper holomorphic embedding of $\mathcal{R}^{\prime}$ to $\mathbb{C}^{2}$.

To conclude the proof of Theorem 1.1 it remains to show that $\overline{\mathcal{R}^{\prime}}$ is diffeomorphic to $\mathcal{R}$. This can be seen as follows. Since $D_{0}$ is a closed simply connected domain with smooth boundary in $U$, there is a smooth function $\rho: \bar{U} \rightarrow \mathbb{R}$ such that $D_{0}=\{\rho \leq 0\}, \rho=1$ on $b U$, and $\rho$ has no critical points in $\bar{U} \backslash C$ (hence $0<\rho(x)<1$ for $\left.x \in U \backslash D_{0}\right)$. For $0 \leq t \leq 1$ set $D_{t}=\{x \in \bar{U}: \rho(x) \leq t\}$; thus $D_{0}$ is the given set and $D_{1}=\bar{U}$. Since $f$ has no critical values in $\bar{U} \backslash C$, the function $\rho \circ f: \mathcal{R} \rightarrow \mathbb{R}$ has no critical points in $\mathcal{R} \backslash f^{-1}(C)$. By Morse theory the set $\mathcal{R}_{t}=\left\{x \in \mathcal{R}: f(x) \in D_{t}\right\}=\{x \in \mathcal{R}: \rho(f(x)) \leq t\}$ is diffeomorphic to $\mathcal{R}=\mathcal{R}_{1}$ for each $t \in[0,1]$. The same is true for $\overline{\mathcal{R}}^{\prime}$ which is a small $\mathcal{C}^{3}$-perturbation of $\mathcal{R}_{0}$. This completes the proof of Theorem 1.1.

\section{Remarks.}

1. In the above proof we could use the Fatou-Bieberbach domains constructed in [Glo] whose boundaries inside a polydisc are small $\mathcal{C}^{1}$-perturbations of the cylinder $b U \times U$; we shall need more smoothness in the proof of Theorem 1.2.

2. To embed $\mathcal{R}$ holomorphically into $\bar{U} \times \mathbb{C}$ using this scheme we would have to find a function $\alpha \in \mathcal{O}(\bar{U})$ whose graph avoids the complex curve $\Sigma \subset \bar{U} \times \mathbb{C}$ constructed above. The fiber $\Sigma_{z}$ over most points $z \in U$ consists of $\left(\begin{array}{l}d \\ 2\end{array}\right)$ points, where $d=\operatorname{deg}(f)$. In the special case when $d=2$ we have $\left(\begin{array}{l}d \\ 2\end{array}\right)=1$ and hence the Oka-principle [Gra, Gro2, FP1, FP2, FP3] applies to sections of $(\bar{U} \times \mathbb{C}) \backslash \Sigma$, so we obtain a desired holomorphic function $\alpha$ whose graph over $\bar{U}$ avoids $\Sigma$. Note that an inner function $f$ of degree $d=2$ exists if and only if $\mathcal{R}$ is hyperelliptic (since we obtain by reflection a degree two meromorphic function on the double $\widehat{\mathcal{R}}$ which implies that $\widehat{\mathcal{R}}$ is hyperelliptic). On the other hand, when $d \geq 3$ the generic fiber of $\Sigma$ contains at least three points and hence its complement is Kobayashi hyperbolic, so the Oka principle does not apply.

The following result shows that there exist complex curves $\Sigma \subset U \times \mathbb{C}$ (not necessarily arising from our construction) which cannot be avoided by holomorphic graphs. On the other hand it is always possible to avoid such a curve by graph of a smooth function; hence the Oka-Grauert principle fails for sections of $(U \times \mathbb{C}) \backslash \Sigma$.

Proposition 2.2. There exists a closed one-dimensional complex subvariety $\Sigma \subset U \times \mathbb{C}$ which does not contain any line $\{z\} \times \mathbb{C}$ and which has a nontrivial intersection with the graph of any holomorphic function on $U$.

Proof. Denote the coordinates on $\mathbb{C}^{2}$ by $(z, w)$. Let $\Sigma_{k} \subset U \times \mathbb{C}$ be the union of the following complex curves, intersected with $U \times \mathbb{C}$ :

$$
z w=1, \quad w=1, \quad w=j z \quad(0 \leq j \leq k) .
$$

Assume that for each $k \in \mathbb{N}$ there is a holomorphic function $\alpha_{k}$ on $U$ whose graph avoids $\Sigma_{k}$. Then $\alpha_{k}$ omits the values 0 and 1 and hence the sequence $\left\{\alpha_{k}\right\}_{k \in \mathbb{N}}$ 
is a normal family on $U$. Passing to a subsequence we may assume that $\alpha_{k}$ converges, uniformly on compacts in $U$, to a holomorphic function $\alpha: U \rightarrow \mathbb{C}$ or to $\alpha=\infty$.

Consider the first case. Choose numbers $0<r<1$ and $k_{0} \in \mathbb{N}$ such that

$$
k_{0} r>\max _{|z|=r} \alpha(z) .
$$

For each $k \geq k_{0}$ the winding number of the function $h_{k}(z)=k z-\alpha(z)$ on the circle $|z|=r$ equals to that of $k z$ which is one. This means that $h_{k}$ has a zero on the disc $r U=\{|z|<r\}$, i.e., the graph of $\alpha$ intersects the line $w=k z$ and hence $\Sigma_{k}$. The same argument holds for any function satisfying (2.2). Since for large $k \in \mathbb{N}$ the function $\alpha_{k}$ is close to $\alpha$ on $r \bar{U}$, its graph also intersects $\Sigma_{k}$, a contradiction.

In the second case when $\alpha_{k} \rightarrow \infty$ we can apply a similar argument in $U \times \overline{\mathbb{C}}$, where $\overline{\mathbb{C}}=\mathbb{C} \cup\{\infty\}$ is the Riemann sphere, to show that for all sufficiently large $k$ the graph of $\alpha_{k}$ intersects the hyperbola $z w=1$ and hence $\Sigma_{k}$, a contradiction. This proves Proposition 2.2.

Remark. The above approach to construct a function $g$ separating points on the fibers of a given inner function $f$ is not quite as ad-hoc as it may seem. Denote by $\mathcal{O}$ the sheaf of germs of holomorphic functions on $\mathcal{R}$. According to Grauert [Gra] the push-forward $f_{*} \mathcal{O}$ is a coherent analytic sheaf of $\mathcal{O}_{U}$-modules over the disc $U=f(\stackrel{\mathcal{R}}{)}$. For each open set $V \subset U$ we may view holomorphic functions on $f^{-1}(V) \subset \mathcal{R}$ as sections of $f_{*} \mathcal{O}$ over $V$. By Cartan's Theorem A [GR] the sheaf $f_{*} \mathcal{O}$ is finitely generated over each compact $K \subset \subset U$, meaning that there exist functions $g_{1}, \ldots, g_{n} \in \mathcal{O}(\stackrel{\circ}{\mathcal{R}})$ such that any $g \in \mathcal{O}(\stackrel{\mathcal{R}}{)}$ may be written in the form $g(x)=\sum_{j=1}^{n} \alpha_{j}(f(x)) \cdot g_{j}(x)\left(x \in f^{-1}(K)\right)$ for some holomorphic functions $\alpha_{1}, \ldots, \alpha_{n}$ defined in a neighborhood of $K$. Now $g$ separates points on the fibers $f^{-1}(z)$ for $z \in K$ if and only if the graph of $\alpha=\left(\alpha_{1}, \ldots, \alpha_{n}\right): K \rightarrow \mathbb{C}^{n}$ avoids a complex hypersurface $\Sigma \subset U \times \mathbb{C}^{n}$ constructed as above. Proposition 2.2 indicates that this may not be possible in general (although we do not have a specific counterexample).

\section{Holomorphic perturbations of bordered Riemann surfaces}

In this section we prove Theorem 1.2. Let $P=(2 U) \times U \subset \mathbb{C}^{2}$. For any sufficiently small perturbation $S$ of the cylinder $b U \times \mathbb{C}$ we denote by $\Omega_{S}$ the connected domain in $P$ bounded by $S$ and containing the origin.

Proposition 3.1. Let $\mathcal{R}$ be a bordered Riemann surface of genus $g_{\mathcal{R}}$ bounded by $m_{\mathcal{R}}$ smooth curves and let $F_{0}=\left(f_{0}, g_{0}\right): \mathcal{R} \rightarrow \bar{U} \times U$ be a map of class $\mathcal{A}^{2}(\mathcal{R})$ such that $f_{0}$ is an inner function on $\mathcal{R}$ with $\operatorname{deg}\left(f_{0}\right) \geq 2 g_{\mathcal{R}}+m_{\mathcal{R}}-1$. For any sufficiently small $\mathcal{C}^{3}$-perturbation $S$ of $S_{0}=b U \times \mathbb{C}$ there is a function $f \in \mathcal{A}^{1}(\mathcal{R})$ which is $\mathcal{C}^{1}$-close to $f_{0}$ such that the map $F=\left(f, g_{0}\right): \mathcal{R} \rightarrow \mathbb{C}^{2}$ satisfies $F(\mathcal{R}) \subset \Omega_{S}$ and $F(b \mathcal{R}) \subset S$. 
We emphasize that the maps $F$ and $F_{0}$ only differ in the first component. If $F_{0}$ is an embedding, it follows that $F$ is also an embedding provided that $S$ is sufficiently $\mathcal{C}^{3}$-close to $b U \times U$.

Assuming Proposition 3.1 for a moment, we now prove Theorem 1.2.

Proof of Theorem 1.2. Let $F_{0}=\left(f_{0}, g_{0}\right): \mathcal{R} \rightarrow \bar{U} \times \mathbb{C}$ satisfy the hypothesis of Theorem 1.2. We may assume that $\mathcal{R}$ is a domain with smooth real-analytic boundary in a larger Riemann surface $\widetilde{\mathcal{R}}$ (section 1 ). Since $f_{0}$ maps $b \mathcal{R}$ to the circle $b U$, it extends by reflection to a holomorphic function in a neighborhood of $\mathcal{R}$. Furthermore we can approximate $g_{0}$ in the $\mathcal{C}^{1}(\mathcal{R})$-sense by a function (still denoted $g_{0}$ ) which is holomorphic in a neighborhood of $\mathcal{R}$. If the approximation is sufficiently close, the new map $F_{0}$ is an embedding in a neighborhood of $\mathcal{R}$. We may assume that $\left\|g_{0}\right\|_{\mathcal{R}}<1$.

Choose a hypersurface $S$ close to $S_{0}=b U \times U$ and the associated domain $\Omega=\Omega_{S} \subset P$ as in Proposition 2.1, with the corresponding injective holomorphic map $\phi: \Omega \rightarrow \mathbb{C}^{2}$ which maps sequences in $\Omega_{S}$ converging to $S$ to sequences going to infinity. Let $F=\left(f, g_{0}\right)$ be a map furnished by Proposition 3.1 which is $\mathcal{C}^{1}$ close to $F_{0}$. If the approximation is sufficiently close then $F$ is a holomorphic

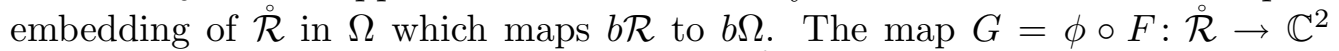
is then a proper holomorphic embedding of $\mathcal{R}$ to $\mathbb{C}^{2}$. This proves Theorem 1.2 granted that Proposition 3.1 is correct.

In the proof of Proposition 3.1 we shall use some results about the linear Riemann-Hilbert problem on bordered Riemann surfaces. Fix a number $0<$ $\alpha<1$. Denote by $\mathcal{A}^{1, \alpha}(b \mathcal{R})$ the Banach algebra of $\mathcal{C}^{1, \alpha}$-functions on $b \mathcal{R}$ which extend holomorphically to $\mathcal{R}$. (The $\mathcal{C}^{1, \alpha}$-norm on $b \mathcal{R}$ can be defined by choosing a smooth parametrization of each curve $C \subset b \mathcal{R}$ by the circle $S^{1}$ and pulling back functions on $C$ to functions on $S^{1}$.)

Given functions $a: b \mathcal{R} \rightarrow \mathbb{C} \backslash\{0\}$ and $c: b \mathcal{R} \rightarrow \mathbb{R}$ of class $\mathcal{C}^{1, \alpha}$, the corresponding Riemann-Hilbert problem is to find $k \in \mathcal{A}^{1, \alpha}(b \mathcal{R})$ such that

$$
\operatorname{Re}(\overline{a(x)} \cdot k(x))=c(x), \quad x \in b \mathcal{R} .
$$

The existence of solutions depends on the index $\kappa(a)$ which is defined as the sum of the winding numbers of $a$ over all $m_{\mathcal{R}}$ boundary components of $\mathcal{R}$ (the corresponding Maslov index is $2 \kappa(a))$. Here we equip $\mathcal{R}$ with the usual orientation induced by the complex structure and we orient the boundary $b \mathcal{R}$ coherently. Note that when $a$ is an inner function on $\mathcal{R}$ we have $\kappa(a)=\operatorname{deg}(a)$. The following is a part of the theorem from [Kop, p. 30]; it corresponds to the case when $\nu=0$ (since we are dealing with functions) and the trivial divisor $\delta$ with degree $n_{\delta}=0$. Notice also that, in [Kop], the surface has $m+1$ holes.

Theorem. Let $\mathcal{R}$ be a bordered Riemann surface of genus $g_{\mathcal{R}}$ with $m_{\mathcal{R}}$ boundary components and let $a$ be a complex-valued Hölder continuous function on $b \mathcal{R}$ without zeros. If $\kappa(a) \geq 2 g_{\mathcal{R}}+m_{\mathcal{R}}-1$ then the Riemann-Hilbert boundary value problem (3.1) is solvable for all Hölder continuous functions $c$ on $b \mathcal{R}$ and 
the corresponding homogeneous problem $(c=0)$ has $2 \kappa(a)-\left(2 g_{\mathcal{R}}+m_{\mathcal{R}}-2\right)$ linearly independent solutions.

\section{Remarks.}

1. Even though the theorem in $[\mathrm{Kop}]$ is stated for the $\mathcal{C}^{\alpha}$-case (the functions $a$ and $c$ are assumed to be of class $\mathcal{C}^{\alpha}$ and the solutions belong to $\mathcal{A}^{\alpha}(b \mathcal{R})$ ), the proof carries over to the case stated here.

2. This is essentially a result concerning the solutions of the operator $L=\bar{\partial}$ acting on sections of the trivial line bundle $E=\mathcal{R} \times \mathbb{C} \rightarrow \mathcal{R}$, with the RiemannHilbert boundary conditions described above. The operator $L$ is elliptic and hence Fredholm, with the (real) index

$$
\operatorname{Ind}(L)=2 \kappa(a)-\left(2 g_{\mathcal{R}}+m_{\mathcal{R}}-2\right) .
$$

Koppelman's theorem asserts that, when $\kappa(a) \geq 2 g_{\mathcal{R}}+m_{\mathcal{R}}-1, L$ is surjective and $\operatorname{dim}_{\mathbb{R}}(\operatorname{ker} L)=\operatorname{Ind}(L)$. For an extension to more general $\bar{\partial}$-type operators we refer to [Gro1] and [HLS].

3. There is a connection (by doubling of $\mathcal{R}$ ) between Koppelman's theorem and the Riemann-Roch theorem [HLS]. The result may be viewed as a special case of the Atiyah-Singer index theorem [AS] which expresses the index of any elliptic linear differential operator, acting on sections of a complex vector bundle $E \rightarrow X$ over a compact manifold $X$, in terms of the Chern class of the bundle and the cohomology class in $H^{*}(X, \mathbb{C})$ determined by the principal symbol of $L$.

Proof of Proposition 3.1. Let $F_{0}=\left(f_{0}, g_{0}\right)$ be as in the proposition. Assume that $\left\|g_{0}\right\|_{\mathcal{R}}<1$ so that $F_{0}(\mathcal{R}) \subset P=(2 U) \times U$. Denote the coordinates on $\mathbb{C}^{2}$ by $(z, w)$. Set $\rho_{0}(z, w)=|z|^{2}-1$. Then $\left\{\rho_{0}=0\right\} \cap P=b U \times U$ and $\rho_{0}\left(f_{0}, g_{0}\right)=0$. For any function $\rho \in \mathcal{C}^{3}(P)$ sufficiently close to $\rho_{0}$ the set $S_{\rho}=\{\rho=0\} \cap P$ is a $\mathcal{C}^{3}$-hypersurface close to $b U \times U$ and vice versa, any small $\mathcal{C}^{3}$-perturbation of $b U \times \mathbb{C}$ within $P$ equals $S_{\rho}$ for some $\rho \in \mathcal{C}^{3}(P)$ close to $\rho_{0}$.

To solve the problem it suffices to find for each $\rho \in \mathcal{C}^{3}(P)$ close to $\rho_{0}$ a function $f=f_{\rho} \in \mathcal{A}^{1, \alpha}(b \mathcal{R})$ close to $f_{0}$ such that $\rho\left(f(x), g_{0}(x)\right)=0$ for all $x \in b \mathcal{R}$. Such $f$ extends from $b \mathcal{R}$ to a function $f \in \mathcal{A}^{1, \alpha}(\mathcal{R})$. The corresponding map $F=\left(f, g_{0}\right): \mathcal{R} \rightarrow P$ takes $b \mathcal{R}$ to $S_{\rho}=\{\rho=0\}$ and it maps the interior $\mathcal{R}$ to the domain $\Omega_{\rho} \subset P$ bounded by $S_{\rho}$ and by $|w|=1$ (for the last statement we need $f$ to be $\mathcal{C}^{1}$-close to $f_{0}$; see [CG] for the details of this argument).

To find such $f=f_{\rho}$ we shall apply the implicit mapping theorem in Banach spaces. Let $D=\left\{f \in \mathcal{A}^{1, \alpha}(b \mathcal{R}):\|f\|<2\right\}$, where $\|$.$\| is the norm on \mathcal{A}^{1, \alpha}(b \mathcal{R})$. We define a Banach space operator

$$
\Phi: \mathcal{C}^{3}(P) \times D \rightarrow \mathcal{C}_{\mathbb{R}}^{1, \alpha}(b \mathcal{R}), \quad \Phi(\rho, f)(x)=\rho\left(f(x), g_{0}(x)\right) \quad(x \in b \mathcal{R}) .
$$

We claim that $\Phi$ is of class $\mathcal{C}^{1}$. Clearly $\Phi$ is linear with respect to $\rho$ and hence $D_{\rho} \Phi(\rho, f)(\tau)=\tau\left(f, g_{0}\right)$. Moreover, Lemma 5.1 in $[\mathrm{HT}]$ implies that for each fixed $\rho \in \mathcal{C}^{3}(P)$ the mapping $f \in \mathcal{A}^{1, \alpha}(b \mathcal{R}) \rightarrow \Phi(\rho, f) \in \mathcal{C}_{\mathbb{R}}^{1, \alpha}(b \mathcal{R})$ is of class $\mathcal{C}^{1}$ and its partial derivative on $f$ equals

$$
D_{f} \Phi(\rho, f)(k)(x)=\operatorname{Re}\left(2 \partial_{z} \rho\left(f(x), g_{0}(x)\right) \cdot k(x)\right), \quad x \in b \mathcal{R} .
$$


It is easily seen that $\Phi$ and its first order partial derivatives are continuous with respect to both variables $(\rho, f)$ and hence $\Phi$ is of class $\mathcal{C}^{1}$ [Lan]. Writing $a_{\rho, f}(x)=2 \partial_{\bar{z}} \rho\left(f(x), g_{0}(x)\right)$ we have

$$
D_{f} \Phi(\rho, f)(k)(x)=\operatorname{Re}\left(\overline{a_{\rho, f}(x)} \cdot k(x)\right), \quad k \in \mathcal{A}^{1, \alpha}(b \mathcal{R}), x \in b \mathcal{R} .
$$

For $\rho_{0}=|z|^{2}-1$ we have $\partial_{\bar{z}} \rho_{0}=z$ and hence $a_{\rho_{0}, f_{0}}=2 f_{0}$ which is nowhere vanishing on $b \mathcal{R}$. Its index equals

$$
\kappa\left(a_{\rho_{0}, f_{0}}\right)=\operatorname{deg}\left(f_{0}\right) \geq 2 g_{\mathcal{R}}+m_{\mathcal{R}}-1 .
$$

Hence the theorem quoted above applies and shows that the linear operator $A=D_{f} \Phi\left(\rho_{0}, f_{0}\right): \mathcal{A}^{1, \alpha}(b \mathcal{R}) \rightarrow \mathcal{C}_{\mathbb{R}}^{1, \alpha}(b \mathcal{R})$ is surjective, with kernel of dimension

$$
\operatorname{dim}_{\mathbb{R}}(\operatorname{ker} A)=2 \operatorname{deg}\left(f_{0}\right)-\left(2 g_{\mathcal{R}}+m_{\mathcal{R}}-2\right) .
$$

Since each finite dimensional subspace in a Banach space is complemented $[\mathrm{Ru} 2$, Lemma 4.21], there is a closed subspace $\mathcal{B}$ in $\mathcal{A}^{1, \alpha}(b \mathcal{R})$ such that $\mathcal{A}^{1, \alpha}(b \mathcal{R})=$ $(\operatorname{ker} A) \oplus \mathcal{B}$ and $A$ maps $\mathcal{B}$ isomorphically onto $\mathcal{C}_{\mathbb{R}}^{1, \alpha}(b \mathcal{R})$. By the implicit function theorem in Banach spaces [Ca] the solutions of the equation $\Phi(\rho, f)=0$ in a small neighborhood of $\left(\rho_{0}, f_{0}\right) \in \mathcal{C}^{3}(P) \times \mathcal{A}^{1, \alpha}(b \mathcal{R})$ are of the form $f(\rho, t)=$ $f_{0}+(t, \varphi(\rho, t))$, where $t \in \operatorname{ker} A$ and $\varphi$ is a $\mathcal{C}^{1}$-operator with image in $\mathcal{B}$ such that $\varphi\left(\rho_{0}, 0\right)=0$ (and hence $\left.f\left(\rho_{0}, 0\right)=f_{0}\right)$. Setting $t=0$ we obtain functions $f_{\rho}=f(\rho, 0) \in \mathcal{A}^{1, \alpha}(b \mathcal{R})$ for $\rho \in \mathcal{C}^{3}(P)$ near $\rho_{0}$ such that $f_{\rho}$ depends differentiably on $\rho$ and satisfies $\rho\left(f_{\rho}, g_{0}\right)=0$ on $b \mathcal{R}$. This concludes the proof of Proposition 3.1.

\section{Families of inner functions on bordered Riemann surfaces}

In this section we prove Theorem 1.4. The essential ingredient is the following result which is possibly of independent interest. We use the notation established in section 1. If $J$ is a complex structure of class $\mathcal{C}^{k-1, \alpha}$ on $\mathcal{R}$, we denote by $\mathcal{A}^{k, \alpha}(\mathcal{R}, J)$ the space of all $J$-holomorphic functions of order $\mathcal{C}^{k, \alpha}$ (that is, their derivatives of order $k$ are Hölder continuous of order $\alpha$ ).

Proposition 4.1. Let $\mathcal{R}$ be a smooth bordered surface of genus $g_{\mathcal{R}}$ and with $m \geq 1$ boundary components. Fix $\alpha \in(0,1)$. Let $J_{0} \in \mathcal{J}_{\mathcal{R}}^{\alpha}$ be a complex structure on $\mathcal{R}$ and let $f_{0} \in \mathcal{A}^{1, \alpha}\left(\mathcal{R}, J_{0}\right)$ be an inner function on $\left(\mathcal{R}, J_{0}\right)$ with multiplicity $\geq 2 g_{\mathcal{R}}+m-1$. Then for each $J \in \mathcal{J}_{\mathcal{R}}^{\alpha}$ sufficiently close to $J_{0}$ there is an inner function $f_{J} \in \mathcal{A}^{1, \alpha}(\mathcal{R}, J)$ near $f_{0}$, with $f_{J}$ depending continuously on $J$ and $f_{J_{0}}=f_{0}$.

Remark. As already mentioned, Ahlfors [Ahl] constructed inner functions of multiplicity $2 g_{\mathcal{R}}+m$ on any bordered Riemann surface. Proposition 4.1 shows that such functions are stable under small perturbations of the complex structure. On the other hand this need not be true for the Ahlfors function $f_{p}$ which maximizes the derivative at a given point $p \in \mathcal{R}$ since the degree of $f_{p}$ may depend on $p$.

Assuming Proposition 4.1 for a moment we can prove Theorem 1.4 as follows. 
Proof. Fix a number $0<\alpha<1$ and let $F_{0}=\left(f_{0}, g_{0}\right): \mathcal{R} \rightarrow \bar{U} \times \mathbb{C}$ be an embedding of class $\mathcal{C}^{1, \alpha}$ which is $J_{0}$-holomorphic on $\mathcal{R}$ for some complex structure $J_{0} \in \mathcal{J}_{\mathcal{R}}^{\alpha}$. Thus $f_{0}$ is an inner function on $\left(\mathcal{R}, J_{0}\right)$ of multiplicity $\geq 2 g_{\mathcal{R}}+m-1$. For each $J \in \mathcal{J}_{\mathcal{R}}^{\alpha}$ sufficiently near $J_{0}$ Proposition 4.1 provides an inner function $f_{J}$ on $(\mathcal{R}, J)$ which is $\mathcal{C}^{1}$-close to $f_{0}$. We can also approximate $g_{0}$ in the $\mathcal{C}^{1}$-sense by $J$-holomorphic functions $g_{J}$ (this is trivial since there is no boundary condition on $g_{J}$ ). If the approximations are sufficiently close (which is the case when $J$ is close enough to $\left.J_{0}\right)$, the $J$-holomorphic map $F_{J}=\left(f_{J}, g_{J}\right): \mathcal{R} \rightarrow \bar{U} \times \mathbb{C}$ is $\mathcal{C}^{1}$-close to $F_{0}$ and hence is an embedding. Hence the Riemann surface $(\mathcal{R}, J)$ is of class $\mathcal{F}$ for all $J$ sufficiently close to $J_{0}$. This proves Theorem 1.4 provided that Proposition 4.1 is correct.

Proof of Proposition 4.1. For each complex structure $J \in \mathcal{J}_{\mathcal{R}}^{\alpha}$ we denote by $\bar{\partial}_{J}$ the corresponding $\bar{\partial}$-operator which maps $\mathcal{C}^{1, \alpha}$-functions on $\mathcal{R}$ to $(0,1)$-forms of class $\mathcal{C}^{\alpha}$ according to the formula

$$
2 \bar{\partial}_{J}(f)=d f+i d f \circ J
$$

Denote the space of such forms by $\Omega_{0,1}^{\alpha}(\mathcal{R}, J)$. Consider the Banach manifolds

$$
\begin{aligned}
\mathcal{W} & =\left\{(f, J): f \in \mathcal{C}^{1, \alpha}(\mathcal{R}),|f|=1 \text { on } b \mathcal{R}, J \in \mathcal{J}_{\mathcal{R}}^{\alpha}\right\}, \\
\mathcal{W}^{0,1} & =\left\{(\omega, J): \omega \in \Omega_{0,1}^{\alpha}(\mathcal{R}, J), J \in \mathcal{J}_{\mathcal{R}}^{\alpha}\right\} .
\end{aligned}
$$

Let $\Phi: \mathcal{W} \rightarrow \mathcal{W}^{0,1}$ be the operator $\Phi(f, J)=\left(2 \bar{\partial}_{J} f, J\right)$. The set

$$
\mathcal{W}^{h}=\left\{(f, J) \in \mathcal{W}: \bar{\partial}_{J}(f)=0\right\}=\{(f, J): \Phi(f, J)=(0, J)\}
$$

consists of $J$-holomorphic inner functions on $\mathcal{R}$ for all complex structures $J$ on $\mathcal{R}$. Denote by $\pi: \mathcal{W} \rightarrow \mathcal{J}_{\mathcal{R}}^{\alpha}$ the projection onto the second factor.

We claim that $\Phi$ is a $\mathcal{C}^{1}$-map of Banach manifolds which is a submersion with finite corank at each point $(f, J) \in \mathcal{W}^{h}$ for which $\kappa(f) \geq 2 g_{\mathcal{R}}+m-1$. Once this is proved, the implicit function theorem [Ca] shows that $\mathcal{W}^{h}$ is a Banach submanifold of $\mathcal{W}$ in a neighborhood of each such point $(f, J)$ and the projection $\pi: \mathcal{W}^{h} \rightarrow \mathcal{J}_{\mathcal{R}}^{\alpha}$ is locally near $(f, J)$ a trivial Banach fibration with finite dimensional fibers. The proposition then follows immediately since it amounts to choosing a local section of this fibration passing through $(f, J)$.

To find the derivative $D \Phi(f, J)(g, K)$ in the direction of a tangent vector $(g, K)$ to $\mathcal{W}$ at $(f, J)$ we choose a local $\mathcal{C}^{1}$ path $\left(f_{t}, J_{t}\right) \in \mathcal{W}$ for $|t|<\epsilon$, with $f_{0}=f, J_{0}=J,\left.\frac{d}{d t}\right|_{t=0} f_{t}=g$ and $\left.\frac{d}{d t}\right|_{t=0} J_{t}=K$. Differentiating the equations $\left|f_{t}\right|^{2}=1$ resp. $J_{t}^{2}=-$ Id with respect to $t$ at $t=0$ we see that $\operatorname{Re}(g \bar{f})=0$ on $b \mathcal{R}$ and $J K+K J=0$. The derivative of $\Phi$ equals

$$
\begin{aligned}
D \Phi(f, J)(g, K) & =\left.\frac{d}{d t}\right|_{t=0} \Phi\left(f_{t}, J_{t}\right) \\
& =(d g+i d g J+i d f K, K) \\
& =\left(2 \bar{\partial}_{J} g+i d f K, K\right) .
\end{aligned}
$$

From this formula we see immediately that $D \Phi(f, J)$ is continuous in $(f, J)$ and hence $\Phi$ is of class $\mathcal{C}^{1}$. Moreover we see that $D \Phi(f, J)$ is surjective if and 
only if any $\omega \in \Omega_{0,1}^{\alpha}(\mathcal{R}, J)$ equals $\omega=2 \bar{\partial}_{J} g+i d f K$ for some $g \in \mathcal{C}^{1, \alpha}(\mathcal{R})$ with $\operatorname{Re}(g \bar{f})=0$ on $b \mathcal{R}$. Since $(i d f K) J=-i d f J K=d f K=-i(i d f K)$ (here we used $K J=-J K$ and $i d f J=-d f)$, the form $i d f K$ is of type $(0,1)$ with respect to $J$. Hence it suffices to see that $g \rightarrow \bar{\partial}_{J} g$ is surjective as a map

$$
\left\{g \in \mathcal{C}^{1, \alpha}(\mathcal{R}): \operatorname{Re}(g \bar{f})=0 \text { on } b \mathcal{R}\right\} \rightarrow \bar{\partial}_{J} g \in \Omega_{0,1}^{\alpha}(\mathcal{R}, J) .
$$

Surjectivity of this map at points $(f, J) \in \mathcal{W}$ with $\kappa(f) \geq 2 g_{\mathcal{R}}+m-1$ is guaranteed by the theorem in [Kop, p. 33] together with Corollary II in [Kop, p. $30]$. (Essentially the result in [Kop] is that we can solve any non-homogeneous Cauchy-Riemann equation on a fixed bordered Riemann surface $(\mathcal{R}, J)$ subject to a Riemann-Hilbert boundary condition, provided that the associated index is sufficiently large, which in our case means that $f$ must have multiplicity $\geq$ $2 g+m-1$.) This shows that $\Phi$ is a submersion at such points as claimed. Furthermore, if $(f, J) \in \mathcal{W}^{h}$, the kernel of the map (4.1) equals

$$
\left\{g \in \mathcal{C}^{1, \alpha}(\mathcal{R}): \bar{\partial}_{J} g=0, \operatorname{Re}(g \bar{f})=0 \text { on } b \mathcal{R}\right\} .
$$

By the cited result in [Kop] this space has dimension $2 \kappa(f)-(2 g+m-2)$. This completes the proof.

\section{Acknowledgements}

We wish to thank Josip Globevnik and Berit Stensønes for stimulating discussions on this topic. This research was supported in part by the Ministry of Science of the Republic of Slovenia.

\section{References}

[Ahl] L. Ahlfors, Open Riemann surfaces and extremal problems on compact subregions, Comment. Math. Helv. 24 (1950), 100-134.

[Ale] H. Alexander, Explicit imbedding of the (punctured) disc into $\mathbb{C}^{2}$, Comment. Math. Helv. 52 (1977), 539-544.

[AS] M.F. Atiyah, I. M. Singer, The index of elliptic operators. I, Ann. of Math. (2) 87 (1968), 484-530.

[BS] H. Behnke, F. Sommer, Theorie der analytischen Funktionen einer komplexen Veränderlichen, Third edition. Springer-Verlag, New York, 1965.

[Ca] H. Cartan, Calcul différentiel, Hermann, Paris, 1967,

[EG] Y. Eliashberg, M. Gromov, Embeddings of Stein manifolds of dimension $n$ into the affine space of dimension 3n/2 + 1, Ann. of Math. (2) 136 (1992), 123-135.

[ČG] M. Černe, J. Globevnik, On holomorphic embedding of planar domains into $\mathbb{C}^{2}$, J. Anal. Math. 81 (2000), 269-282.

[FK] H. M. Farkas, I. Kra, Riemann surfaces, Second edition. Graduate Texts in Mathematics, 71. Springer-Verlag, New York, 1992.

[FP1] F. Forstnerič, J. Prezelj, Oka's principle for holomorphic fiber bundles with sprays, Math. Ann. 317 (2000), 117-154.

[FP2] _ Oka's principle for holomorphic submersions with sprays, Math. Ann. 322 (2002), 633-666.

[FP3] , Extending holomorphic sections from complex subvarieties, Math. Z. 236 (2001), 43-68.

[Glo] J. Globevnik, On Fatou-Bieberbach domains, Math. Z. 229 (1998), 91-106. 
[GS] J. Globevnik, B. Stensønes, Holomorphic embeddings of planar domains into $\mathbb{C}^{2}$, Math. Ann. 303 (1995), 579-597.

[Gou] T. Gouma, Ahlfors functions on non-planar Riemann surfaces whose double are hyperelliptic, J. Math. Soc. Japan 50 (1998), 685-695.

[Gra] H. Grauert, Holomorphe Funktionen mit Werten in komplexen Lieschen Gruppen, Math. Ann. 133 (1957), 450-472.

[Gro1] M. Gromov, Pseudoholomorphic curves in symplectic manifolds, Invent. Math. 82 (1985), 307-347.

[Gro2] Oka's principle for holomorphic sections of elliptic bundles, J. Amer. Math. Soc. 2 (1989), 851-897.

[GR] C. Gunning, H. Rossi, Analytic functions of several complex variables, Prentice-Hall, Englewood Cliffs, NJ, 1965.

[HT] D. C. Hill, D. Taiani, Families of analytic discs in $\mathbb{C}^{n}$ with boundaries in a prescribed CR manifold, Ann. Scuola Norm. Sup. Pisa Cl. Sci. (4) 5 (1978), 327-380.

[HLS] H. Hofer, V. Lizan, J.-C. Sikorav, On genericity for holomorphic curves in fourdimensional almost-complex manifolds, J. Geom. Anal. 7 (1997), 149-159.

[Hör] L. Hörmander, An introduction to complex analysis in several variables, Third edition. North-Holland Mathematical Library, 7. North-Holland Publishing, Amsterdam, 1990.

[Kop] W. Koppelman, The Riemann-Hilbert problem for finite Riemannian surfaces, Comm. Pure Appl. Math. 12 (1959), 13-35.

[Lan] S. Lang, Differential and Riemannian manifolds, Third edition. Graduate Texts in Mathematics, 160. Springer-Verlag, New York, 1995.

[Lau] H. B. Laufer, Imbedding annuli in $\mathbf{C}^{\mathbf{2}}$, J. Analyse Math. 26 (1973), 187-215.

[NN] A. Newlander, L. Nirenberg, Complex analytic coordinates in almost complex manifolds, Ann. of Math. (2) 65 (1957), 391-404.

[Ru1] W. Rudin, Pairs of inner functions on finite Riemann surfaces, Trans. Amer. Math. Soc. 140 (1969), 423-434.

[Ru2] , Functional analysis, McGraw-Hill, New York, 1973.

[Sik] J. C. Sikorav, Proof that every torus with one hole can be properly holomorphically embedded in $\mathbb{C}^{2}$, preprint, October 1997 (unpublished).

[SS] M. Schiffer, D. C. Spencer, Functionals of finite Riemann surfaces, Princeton Univ. Press, Princeton, NJ, 1954.

[Sch] J. Schürmann, Embeddings of Stein spaces into affine spaces of minimal dimension, Math. Ann. 307 (1997), 381-399.

[Spr] G. Springer, Introduction to Riemann surfaces, Addison-Wesley, Reading, MA, 1957.

[Ste] J.-L. Stehlé, Plongements du disque dans $\mathbb{C}^{2}$, Séminaire Pierre Lelong (Analyse), Année 1970-1971, pp. 119-130. Lecture Notes in Math., Vol. 275, Springer, Berlin, 1972.

[Stn] B. Stensønes, Fatou-Bieberbach domains with $\mathcal{C}^{\infty}$-smooth boundary, Ann. of Math. (2) 145 (1997), 365-377.

[Tsa] V.V. Tsanov, On hyperelliptic Riemann surfaces and doubly generated function algebras, C. R. Acad. Bulgare Sci. 31 (1978), 1249-1252.

Institute of Mathematics, Physics and Mechanics, University of Ljubljana, Jadranska 19, 1000 Ljubluana, Slovenia.

E-mail address: Miran.Cerne@fmf.uni-lj.si

E-mail address: Franc.Forstneric@fmf.uni-lj.si 